\title{
21. INTERSTITIAL WATER STUDIES ON SMALL CORE SAMPLES, LEG $14^{1}$
}

\author{
Lee S. Waterman and Frederick L. Sayles, Woods Hole Oceanographic Institution, Woods Hole, Massachusetts \\ Frank T. Manheim, United States Geological Survey, Woods Hole, Massachusetts
}

The interstitial waters from the sediments cored on Leg 14 exhibit characteristic compositional trends with three important exceptions. At most of the sites, the changes in $\mathrm{Na}$ and $\mathrm{Cl}$ are very small while enrichment of $\mathrm{Ca}$ and $\mathrm{Sr}$ and depletion of $\mathrm{Mg}$ and $\mathrm{SO}_{4}$ is typical of patterns observed in similar types of sediment recovered on previous legs. At Sites 139 and 140, off the African coast, and Site 144, off the coast of South America, systematic increases in concentration with depth are found for $\mathrm{Na}$ and $\mathrm{Cl}$. At these sites, the concentration changes, in $\mathrm{Ca}, \mathrm{Mg}, \mathrm{SO}_{4}$ and $\mathrm{Sr}$ generally follow diagenic patterns observed previously, but there is some indication of other factors affecting $\mathrm{Mg}$ concentrations.

All analyses reported here, with the exception of bulk water content and $\mathrm{pH}$, were carried out on water samples which were squeezed from the sediments on board ship and preserved by heat-sealing in lengths of polyethylene tubing. Analytical methods were identical to those outlined in earlier volumes of this series. We wish to thank Wen Chang, James O'Neill and Irene Uhlitzsch for their assistance in conducting the laboratory determinations and data reduction.

\section{RESULTS}

At Sites 135, 137, and 141, potassium concentrations decrease with depth to about half ocean water values (0.14-0.19 \%०) at the limits of sampling. Likewise, increases in calcium and simultaneous decreases in magnesium occur with depth at all four sites. This effect is most evident at Site 137 where the calcium enrichment is eight fold and the magnesium concentration is reduced to about half the ocean water value. At Site $136, \mathrm{~K}$ and $\mathrm{Mg}$ levels are somewhat lower than normal, while $\mathrm{Ca}$ is about twice the ocean-water value. Strontium concentrations increase strongly at Site 135 , weakly at 137, and are irregularly higher than ocean water at Site 141. At Site 136 strontium concentrations decrease from $80 \mathrm{ppm}$ at 136 meters to 33 ppm at 282 meters.

At Site 142, the first 311 meters, comprising the upper reflective zone, is clay-rich continental margin type sediment similar to those recovered on Leg 11. Interstitial solutions exhibit significant depletions of potassium, calcium, magnesium, and sulfate which are characteristic of rapidly deposited, terrigenous derived sediments. Solutions from the interval 380 to 587 meters, by contrast, are characteristic of biogenic sediments with the calcium and magnesium levels increasing to the 0.8 to $1.0 \%$ range. Sulfate, which is close to the threshold of detection in the top interval, increases to about half the concentration of ocean water $(1.42 \%)$ at the bottom of the hole.

\footnotetext{
${ }^{1}$ Contribution Number 2861 of the Woods Hole Oceanographic Institution. Publication approved by the Director, U.S.G.S.
}

The most striking feature of Sites 139,140 , and 144 is the increase in $\mathrm{Na}$ and $\mathrm{Cl}$ with depth (Figure 1). Chlorinities increase to values of 44,32 , and $28 \%$ respectively. Corresponding increases in $\mathrm{Na}$ concentrations occur at all three sites. A single sample (144-5-cc) does not fit the trend. The shipboard salinity determination of 41.8 indicates that the analysis is correct and that the concentration decrease in the sample is real. This reversal of the concentration trend is thought to reflect contamination of the sediment with seawater prior to squeezing.

Depletion of K and enrichment of Ca at Sites 140, and 144 are comparable to the changes noted above for other Leg 14 sites and in earlier initial reports for similar types of sediment. Both $\mathrm{K}$ and $\mathrm{Ca}$ are enriched at Site 139. The depletion of $\mathrm{SO}_{4}$ at all three sites likewise resembles previously observed diagenic patterns. Magnesium is depleted at Sites 140 and 144 , but is slightly enriched at Site 139. The enrichment of $\mathrm{Mg}$ at Site 139 and the increase in $\mathrm{Mg}$ at the bottom of Site 144 are unusual for sediments in which interstitial $\mathrm{SO}_{4}$ has been strongly depleted. Previous experience (Legs $1,4,10,11,12$ ) has led us to expect depletions of $\mathrm{Mg}$ on the order of 50 to 60 per cent of ocean water concentrations in sediments where interstitial $\mathrm{SO}_{4}$ is nearly exhausted with depth. Further, $\mathrm{Mg}$ concentration has been found almost invariably to decrease with increasing depth under conditions of $\mathrm{SO}_{4}$ reduction. The $\mathrm{Sr}$ concentrations at depth at Site 144 are the highest yet reported.

\section{DISCUSSION}

The compositional trends of the type seen in the interstitial water at Sites 136, 137, 138, 141, and 142 have been discussed in previous leg reports. Changes in the composition of pore solutions from primarily biogenic sediments have been considered in the initial reports for Legs $6,7,8$ and 9. Sediments containing considerable amounts of relatively rapidly deposited terrigenous silicate detritus were cored on Legs 10,11, and 12; the chemistry of interstitial solutions of such sediment is discussed in these reports. No attempt will be made in this report to review, or further pursue these discussions.

The concentration gradients of both $\mathrm{Na}$ and $\mathrm{Cl}$ at Sites 139,140 , and 144 indicate that evaporite deposits exist at depth at all three locations. Previously, such $\mathrm{NaCl}$ enrichment has been found only in sediments where evaporites are known or suspected at depth cf initial reports, Legs 1, 10 and 13. While evaporation of the samples would produce $\mathrm{NaCl}$ enrichment, such artifacts could not be expected to produce the consistent concentration depth trends of Figure 1. The concentration of both $\mathrm{Na}$ and $\mathrm{Cl}$ increase fairly smoothly with depth at Sites 140 and 144 . While there is considerable scatter in the data, this does not obscure a similar trend at Site 139. Further, salinity 


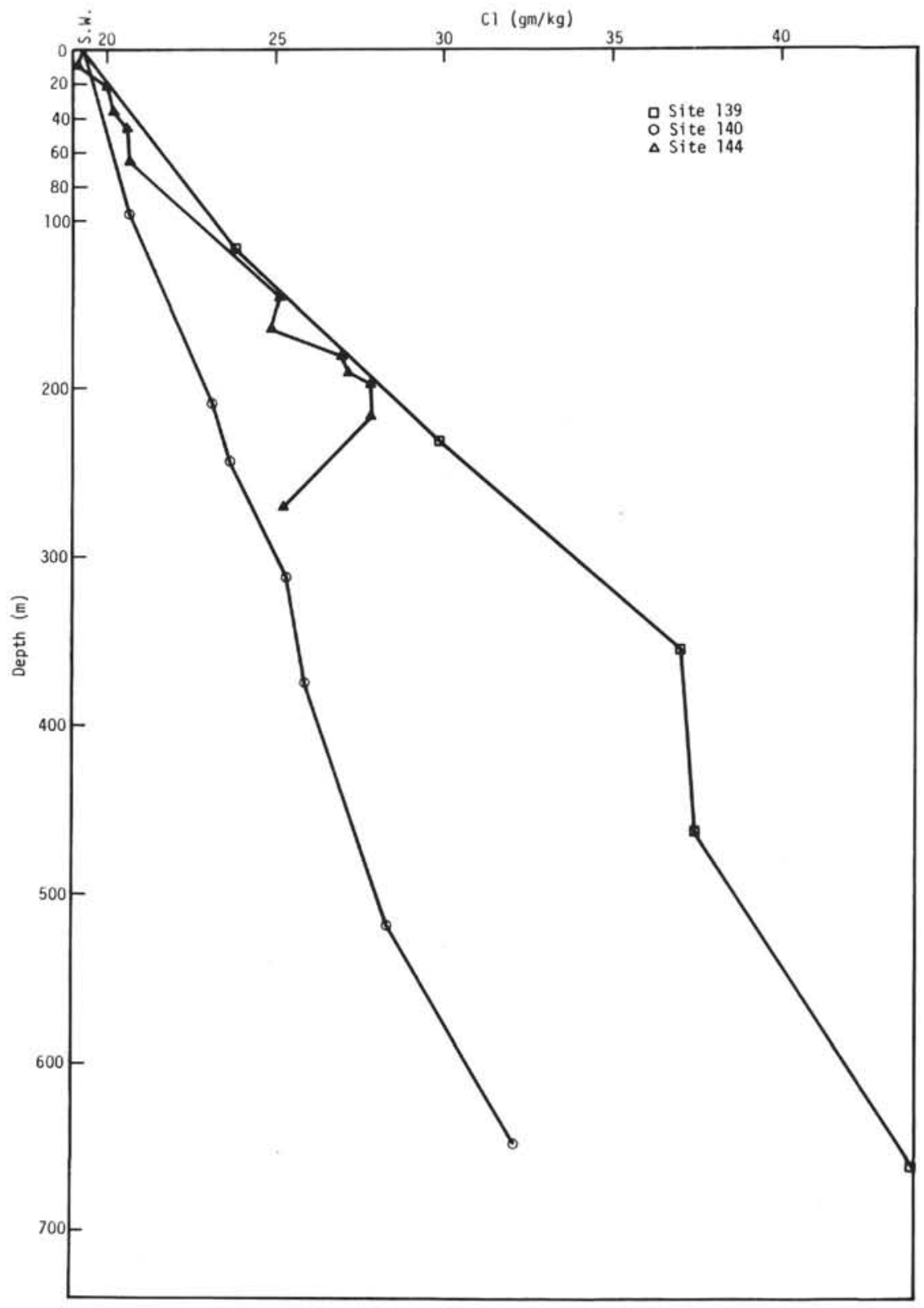

Figure 1. Salinity gradients at Sites 139, 140 and 144.

measurements made on the ship tally well with measurements made in the laboratory thereby ruling out evaporation during storage. Evaporation prior to processing cannot be a factor as sampling of the sediments was done immediately upon recovery of the cores. Similar scatter in $\mathrm{Cl}$ as a function of depth has been reported by Manheim and Bischoff (1969) in deep cores from the Gulf of Mexico. There is little reason, therefore, to doubt that the observed $\mathrm{NaCl}$ enrichments at all three sites result from dissolution of deeply buried evaporites.

The existence and dissolution of evaporites at depth does not appear to have influenced the concentration of $\mathrm{K}$, $\mathrm{Ca}$ and $\mathrm{SO}_{4}$. The concentration changes in these three constituents are similar in direction and in magnitude to changes seen in similar types of sediment where no evaporites exist. $\mathrm{Mg}$, on the other hand, appears to exhibit somewhat unusual behavior. The absence of the usual $\mathrm{Mg}$ depletions accompanying strong $\mathrm{SO}_{4}$ reduction at 139 and the slight increase in $\mathrm{Mg}$ noted at the bottom of 144 may be due to diffusive addition of $\mathrm{Mg}$ along with $\mathrm{Na}$ and $\mathrm{Cl}$.

\section{REFERENCES}

Manheim, F. T. and Bischoff, J. L., 1969. Geochemistry of pore waters from Shell Oil Company drill holes on the continental slope of the Northern Gulf of Mexico. Chem. Geol. 4, 63. 
TABLE 1

Major Constituents of Pore Fluids. Values in $\mathrm{g} / \mathrm{kg}(\%)$ Except as Noted.

\begin{tabular}{|c|c|c|c|c|c|c|c|c|c|c|c|c|c|c|c|c|c|c|}
\hline $\begin{array}{l}\text { Sample } \\
\text { Desig- } \\
\text { nation }\end{array}$ & $\begin{array}{l}\text { Depth } \\
(\mathrm{m})\end{array}$ & Age & Description & $\mathrm{Na}^{\mathrm{a}}$ & $\mathrm{Na}^{\mathrm{b}}$ & $\mathrm{K}$ & $\mathrm{Ca}$ & $\mathrm{Mg}$ & $\begin{array}{c}\text { Total } \\
\text { Cations } \\
\text { (meq } / \mathrm{kg})\end{array}$ & $\mathrm{Cl}$ & $\mathrm{SO}_{4}$ & $\begin{array}{c}\text { Alk. } \\
(\mathrm{meq} / \mathrm{kg})\end{array}$ & $\mathrm{HCO}_{3}{ }^{\mathrm{c}}$ & $\begin{array}{c}\text { Total } \\
\text { Anions } \\
\text { (meq } / \mathrm{kg} \text { ) }\end{array}$ & Sum $^{\mathrm{d}}$ & $\begin{array}{l}\text { Refrac- } \\
\text { tometer }\end{array}$ & $\begin{array}{l}\mathrm{H}_{2} \mathrm{O} \\
(\%\end{array}$ & $\mathrm{pH}$ \\
\hline Hole 135 & $\left(32^{\circ} 20\right.$ & $.8^{\prime} \mathrm{N}, 10^{\circ} \quad 25.5$ & $5^{\prime} \mathrm{W}$, water depth $4152 \mathrm{~m}$ & $\mathrm{~m}$, top & ographi & ic high & SE o & f Horse & shoe Abyss: & l Plain) & & & & & & & & \\
\hline \multicolumn{4}{|c|}{ Surface ocean water } & 11.3 & 11.0 & 0.40 & 0.43 & 1.32 & 617 & 20.16 & 2.90 & 2.7 & 0.16 & 632 & 36.7 & 36.8 & - & - \\
\hline $135-1-2$ & 3 & Pleistocene & $\begin{array}{l}\text { Varicolored light brown- } \\
\text { ish gray nanno chalk ooze. }\end{array}$ & 10.9 & 10.7 & 0.46 & 0.39 & 1.22 & 597 & 19.60 & 2.55 & 2.4 & 0.14 & 608 & 35.3 & 35.5 & 37 & 7.4 \\
\hline $2-6$ & 82 & $\begin{array}{l}\text { Late } \\
\text { Miocene }\end{array}$ & $\begin{array}{l}\text { Light gray nanno chalk } \\
\text { ooze, some black streaks. }\end{array}$ & 10.9 & 10.7 & 0.41 & 0.39 & 1.11 & 587 & 19.62 & 2.02 & 1.4 & 0.08 & 596 & 34.5 & 34.6 & 32 & 7.2 \\
\hline $3-2$ & 176 & $\begin{array}{l}\text { Late } \\
\text { Miocene }\end{array}$ & $\begin{array}{l}\text { Varicolored light gray } \\
\text { nanno chalk ooze. }\end{array}$ & 11.0 & 10.8 & 0.34 & 0.55 & 1.02 & 588 & 19.72 & 1.87 & 2.1 & 0.13 & 597 & 34.6 & 34.4 & 29 & 7.4 \\
\hline $4-2$ & 260 & $\begin{array}{l}\text { Middle } \\
\text { Miocene }\end{array}$ & $\begin{array}{l}\text { Varicolored light gray } \\
\text { nanno chalk ooze. }\end{array}$ & 11.1 & 10.8 & 0.30 & 0.70 & 0.97 & 595 & 20.16 & 1.71 & 2.1 & 0.13 & 607 & 34.1 & 34.6 & 26 & 7.3 \\
\hline $7-3$ & 435 & $\begin{array}{l}\text { Lower } \\
\text { Campanian }\end{array}$ & $\begin{array}{l}\text { Green, olive gray, dark } \\
\text { brown silty mudstone, } \\
\text { sand and "red clay" } \\
\text { layers, intercalated. }\end{array}$ & 10.9 & 10.8 & 0.19 & 0.80 & 0.87 & 584 & 19.99 & 1.25 & 2.2 & 0.13 & 592 & 34.1 & 34.1 & 21 & 7.8 \\
\hline \multicolumn{19}{|c|}{ Hole $136\left(34^{\circ} 10.1^{\prime} \mathrm{N}, 16^{\circ} 18.2^{\prime} \mathrm{W}\right.$, water depth $4169 \mathrm{~m}$} \\
\hline \multicolumn{4}{|c|}{ Surface ocean water } & 11.3 & 11.2 & 0.41 & 0.43 & 1.36 & 632 & 20.44 & 2.72 & 3.2 & 0.20 & 636 & 36.9 & 37.4 & - & - \\
\hline $136-1-5$ & 136 & $\begin{array}{ll}\text { Early } \\
\text { Pliocene }\end{array}$ & $\begin{array}{l}\text { Very pale orange nanno } \\
\text { chalk ooze. }\end{array}$ & 10.8 & 10.6 & 0.29 & 0.90 & 1.01 & 596 & 19.68 & 2.28 & 2.4 & 0.15 & 604 & 35.1 & 35.8 & 27 & 5.4 \\
\hline $2-6$ & 224 & $\begin{array}{l}\text { Middle Early } \\
\text { Miocene }\end{array}$ & $\begin{array}{l}\text { Very pale orange and pale } \\
\text { brown nanno chalk ooze. }\end{array}$ & 11.0 & 10.8 & 0.30 & 0.83 & 1.06 & 605 & 19.95 & 2.39 & 2.0 & 0.12 & 614 & 35.6 & 35.8 & 27 & 6.9 \\
\hline $3-4$ & 238 & $\begin{array}{ll}\text { Early } \\
\text { Miocene }\end{array}$ & $\begin{array}{l}\text { Very pale brown to pale } \\
\text { brown nanno chalk ooze } \\
\text { with light gray lenses and } \\
\text { mottles. }\end{array}$ & 11.0 & 10.6 & 0.29 & 0.83 & 1.02 & 594 & 19.76 & 2.39 & 3.0 & 0.18 & 609 & 35.5 & 35.2 & 24 & 6.8 \\
\hline $4-3$ & 248 & $\begin{array}{l}\text { Early } \\
\text { Miocene (?) }\end{array}$ & $\begin{array}{l}\text { Brown and grayish brown } \\
\text { brown silty clay. }\end{array}$ & 10.8 & 10.6 & 0.28 & 0.83 & 1.06 & 597 & 19.71 & 2.38 & 1.6 & 0.10 & 606 & 35.2 & 35.2 & 32 & 6.9 \\
\hline $6-1$ & 264 & $\begin{array}{l}\text { Santonian to } \mathrm{H} \\
\text { Campanian }\end{array}$ & $\begin{array}{l}\text { Brownish red and pale } \\
\text { yellowish brown silty clay. }\end{array}$ & 10.0 & 9.8 & 0.21 & 0.84 & 1.12 & 564 & 18.58 & 2.31 & 1.9 & 0.11 & 574 & 33.2 & 33.0 & 40 & 6.6 \\
\hline $8-2$ & 282 & Unknown & Reddish brown marl mud. & 10.3 & 10.1 & 0.22 & 0.90 & 1.09 & 578 & 19.12 & 2.36 & 1.0 & 0.06 & 589 & 34.0 & 34.1 & 35 & 6.5 \\
\hline \multicolumn{19}{|c|}{ Hole $137\left(25^{\circ} 55.5^{\prime} \mathrm{N}, 27^{\circ} 03.6^{\prime} \mathrm{W}\right.$, water depth $5361 \mathrm{~m}$} \\
\hline \multicolumn{4}{|c|}{ Surface ocean water } & 11.5 & 11.4 & 0.41 & 0.44 & 1.40 & 642 & 20.76 & 2.90 & 2.8 & 0.17 & 649 & 37.6 & 37.4 & - & - \\
\hline $137-1-5$ & 58 & Unknown & $\begin{array}{l}\text { Yellowish brown and dark } \\
\text { grayish brown silty clay. }\end{array}$ & 10.4 & 10.5 & 0.39 & 0.57 & 1.24 & 608 & 19.40 & 2.57 & 2.6 & 0.16 & 603 & 34.7 & 35.2 & 39 & 6.9 \\
\hline $2-1$ & 100 & Unknown & $\begin{array}{l}\text { Brick red silty zeolitic } \\
\text { clay. }\end{array}$ & 11.1 & 10.8 & 0.40 & 0.66 & 1.20 & 612 & 20.17 & 2.66 & 1.8 & 0.11 & 626 & 36.3 & 36.3 & 57 & 7.1 \\
\hline 2-CC 1 & 104 & Unknown & Brown clay. & 10.7 & 10.6 & 0.38 & 0.74 & 1.19 & 604 & 19.80 & 2.53 & 1.8 & 0.11 & 612 & 35.4 & 35.2 & 57 & 7.0 \\
\hline $3-6 \quad 1$ & 143 & Unknown & $\begin{array}{l}\text { Varicolored brown clay, } \\
\text { slightly zeolitic }\end{array}$ & 10.5 & 10.3 & 0.33 & 0.93 & 1.12 & 593 & 19.69 & 2.21 & 1.3 & 0.08 & 602 & 34.9 & 35.5 & 36 & 6.7 \\
\hline $4-2$ & 168 & Unknown & $\begin{array}{l}\text { Reddish brown clay, } \\
\text { slightly silty, zeolitic. }\end{array}$ & 10.0 & 9.9 & 0.27 & 1.15 & 1.17 & 591 & 19.45 & 2.33 & 1.1 & 0.07 & 598 & 34.4 & 35.2 & 38 & 7.0 \\
\hline 6-CC 2 & 225 & $\begin{array}{l}\text { Turonian-(?) } \\
\text { Coniacian }\end{array}$ & $\begin{array}{l}\text { Reddish brown clay, } \\
\text { zeolitic. }\end{array}$ & 9.9 & 9.6 & 0.23 & 1.62 & 1.04 & 591 & 19.66 & 2.31 & 0.8 & 0.05 & 603 & 34.8 & 35.8 & 36 & 6.7 \\
\hline
\end{tabular}


TABLE 1 - Continued

\begin{tabular}{|c|c|c|c|c|c|c|c|c|c|c|c|c|c|c|c|c|c|c|}
\hline $\begin{array}{l}\text { Sample } \\
\text { Desig- } \\
\text { nation }\end{array}$ & $\begin{array}{c}\text { Depth } \\
\text { (m) }\end{array}$ & Age & Description & $\mathrm{Na}^{\mathrm{a}}$ & $\mathrm{Na}^{\mathrm{b}}$ & K & $\mathrm{Ca}$ & $\mathrm{Mg}$ & $\begin{array}{c}\text { Total } \\
\text { Cations } \\
(\mathrm{meq} / \mathrm{kg})\end{array}$ & $\mathrm{Cl}$ & $\mathrm{SO}_{4}$ & $\underset{(\mathrm{meq} / \mathrm{kg})}{\text { Alk. }}$ & $\mathrm{HCO}_{3}{ }^{\mathrm{C}}$ & $\begin{array}{c}\text { Total } \\
\text { Anions } \\
\text { (meq } / \mathrm{kg})\end{array}$ & Sum $^{d}$ & $\begin{array}{l}\text { Refrac- } \\
\text { tometer }\end{array}$ & $\begin{array}{l}\mathrm{H}_{2} \mathrm{O} \\
(\% \%)^{\mathrm{e}}\end{array}$ & $\mathrm{pH}$ \\
\hline $137-7-\mathrm{CC}$ & 265 & $\begin{array}{l}\text { Cenomanian } \\
\text {-Early } \\
\text { Turonian }\end{array}$ & $\begin{array}{l}\text { Varicolored alternations of } \\
\text { dark carbonaceous clay, } \\
\text { nanno marl ooze and nanno } \\
\text { chalk ooze. }\end{array}$ & 9.7 & 9.6 & 0.22 & 2.28 & 0.76 & 601 & 19.99 & 1.91 & 0.8 & 0.05 & 604 & 34.9 & 36.0 & 33 & - \\
\hline $8-\mathrm{CC}$ & 274 & $\begin{array}{l}\text { Late } \\
\text { Cenomanian }\end{array}$ & $\begin{array}{l}\text { Dark gray foram-nanno } \\
\text { chalk ooze. }\end{array}$ & 10.0 & 9.8 & 0.24 & 2.16 & 0.82 & 608 & 20.23 & 2.01 & 2.8 & 0.17 & 615 & 35.6 & 36.3 & 32 & 7.0 \\
\hline $9-5$ & 282 & $\begin{array}{l}\text { Late } \\
\text { Cenomanian }\end{array}$ & $\begin{array}{l}\text { Pale red and light green- } \\
\text { ish gray nanno marl ooze } \\
\text { to chalk ooze. }\end{array}$ & 9.5 & 9.6 & 0.19 & 2.34 & 0.79 & 602 & 19.88 & 1.83 & 1.2 & 0.07 & 600 & 34.6 & 35.8 & 26 & 6.0 \\
\hline $10-5$ & 287 & Cenomanian & $\begin{array}{l}\text { Reddish brown and } \\
\text { greenish gray nanno marl } \\
\text { to chalk ooze. }\end{array}$ & 9.5 & 9.4 & 0.19 & 2.51 & 0.76 & 605 & 20.09 & 1.86 & 1.1 & 0.07 & 606 & 35.0 & 36.3 & 27 & 6.4 \\
\hline $16-5$ & 381 & $\begin{array}{l}\text { Late } \\
\text { Albian }\end{array}$ & $\begin{array}{l}\text { Gray and brown nanno } \\
\text { marl ooze and foram- } \\
\text { nanno chalk ooze. }\end{array}$ & 9.2 & 9.2 & 0.14 & 3.20 & 0.68 & 622 & 20.47 & 1.92 & 0.5 & 0.03 & 618 & 35.6 & 37.4 & 27 & 5.7 \\
\hline
\end{tabular}

\section{Hole $138\left(25^{\circ} 55.4^{\prime} \mathrm{N}, 25^{\circ} 33.8^{\prime} \mathrm{W}\right.$, water depth $5288 \mathrm{~m}$, foot of continental rise $870 \mathrm{Km}$. W of Cap Blanc)}

\begin{tabular}{|c|c|c|c|c|c|c|c|c|c|c|c|c|c|c|c|c|c|c|}
\hline $138-1-5$ & 58 & $\begin{array}{l}\text { Early } \\
\text { Miocene }\end{array}$ & $\begin{array}{l}\text { Pale yellowish green and } \\
\text { pale yellowish brown clay } \\
\text { and sand. }\end{array}$ & 10.9 & 10.7 & 0.40 & 0.46 & 1.26 & 604 & 19.71 & 2.49 & 2.7 & 0.17 & 612 & 35.4 & 35.2 & 62 & - \\
\hline $2-5$ & 116 & Oligocene & $\begin{array}{l}\text { Greenish gray and olive } \\
\text { gray silty clay. }\end{array}$ & 10.4 & 10.3 & 0.33 & 0.54 & 1.24 & 585 & 19.25 & 2.09 & 4.5 & 0.27 & 590 & 34.1 & 35.2 & 47 & - \\
\hline Hole 139 & $\left(23^{\circ}\right.$ & $31.1^{\prime} \mathrm{N}, 18^{\circ}$ & $42.3^{\prime} \mathrm{W}$, water depth 3047 & $\mathrm{~m}, \mathrm{mi}$ & Idle ce & ontinent & tal rise & 250 & $\mathrm{Km}$. W of & Cap Blanc) & & & & & & & & \\
\hline $139-1-2$ & 116 & $\begin{array}{l}\text { Early } \\
\text { Pliocene }\end{array}$ & $\begin{array}{l}\text { Light olive gray to light } \\
\text { gray foram nanno chalk } \\
\text { ooze; } \mathrm{H}_{2} \mathrm{~S} \text { odor }\end{array}$ & 13.1 & 12.9 & 0.47 & 0.29 & 1.07 & 674 & 23.78 & 0.22 & 10.2 & 0.62 & 686 & 39.6 & 39.6 & 37 & 7.3 \\
\hline $2-4$ & 229 & $\begin{array}{l}\text { Early } \\
\text { Pliocene }\end{array}$ & $\begin{array}{l}\text { Light gray foram nanno } \\
\text { chalk ooze. }\end{array}$ & 16.1 & 15.8 & 0.49 & 0.59 & 1.25 & 830 & 29.87 & $<.01$ & 4.4 & 0.27 & 847 & 48.6 & 49.5 & 31 & 7.3 \\
\hline $3-\mathrm{CC}$ & 354 & $\begin{array}{l}\text { Middle } \\
\text { Miocene }\end{array}$ & $\begin{array}{l}\text { Greenish gray nanno } \\
\text { marl chalk ooze. }\end{array}$ & 20.0 & 19.4 & 0.61 & 1.03 & 1.39 & 1022 & 37.14 & $<.01$ & 1.0 & 0.06 & 1049 & 60.2 & 61.6 & - & 7.1 \\
\hline 4-CC & 463 & $\begin{array}{l}\text { Middle } \\
\text { Miocene }\end{array}$ & $\begin{array}{l}\text { Greenish gray nanno } \\
\text { marl ooze. }\end{array}$ & 20.0 & 19.8 & 0.56 & 1.36 & 1.37 & 1058 & 37.51 & 0.11 & 3.1 & 0.19 & 1063 & 61.1 & 62.2 & - & 7.1 \\
\hline $7-3$ & 659 & $\begin{array}{l}\text { Early } \\
\text { Miocene }\end{array}$ & $\begin{array}{l}\text { Olive to olive gray quartz } \\
\text { sand and diatom mud. }\end{array}$ & 23.3 & 24.0 & 0.53 & 2.15 & 1.31 & 1278 & 43.99 & 0.08 & 0.8 & 0.05 & 1242 & 74.1 & 78.1 & 28 & 7.1 \\
\hline
\end{tabular}

Holes 140 and $140 \mathrm{~A}\left(21^{\circ} 45.0^{\prime} \mathrm{N}, 21^{\circ} 47.5^{\prime} \mathrm{W}\right.$, water depth $4483 \mathrm{~m}$, foot of continental rise $450 \mathrm{~km} \mathrm{~W}$ of Cap Blanc)

\begin{tabular}{|c|c|c|c|c|c|c|c|c|c|c|c|c|c|c|c|c|c|c|}
\hline $140-1-6$ & 96 & $\begin{array}{l}\text { Late } \\
\text { Pliocene }\end{array}$ & $\begin{array}{l}\text { Very light yellowish gray } \\
\text { foram-nanno chalk ooze. }\end{array}$ & 11.4 & 11.4 & 0.43 & 0.34 & 1.27 & 627 & 20.66 & 2.07 & 4.0 & 0.24 & 630 & 36.4 & 36.9 & 39 & 7.4 \\
\hline $2-6$ & 209 & $\begin{array}{l}\text { Early } \\
\text { Miocene }\end{array}$ & $\begin{array}{l}\text { Grayish olive diatom } \\
\text { mud/ooze, quartz bearing. }\end{array}$ & 12.8 & 12.5 & 0.39 & 0.65 & 1.18 & 684 & 23.16 & 1.89 & 2.3 & 0.14 & 695 & 40.2 & 40.2 & 43 & 7.5 \\
\hline $140 \mathrm{~A}-2-6$ & 243 & $\begin{array}{l}\text { Middle } \\
\text { Eocene }\end{array}$ & $\begin{array}{l}\text { Varicolored green and } \\
\text { gray clay, slightly } \\
\text { siliceous, firm }\end{array}$ & 12.6 & 12.3 & 0.28 & 0.79 & 1.20 & 680 & 23.66 & 1.08 & 3.2 & 0.20 & 693 & 39.8 & 40.7 & 38 & 7.5 \\
\hline $140-3-2$ & 313 & $\begin{array}{l}\text { Middle } \\
\text { Eocene }\end{array}$ & $\begin{array}{l}\text { Greenish gray silty clay } \\
\text { slightly siliceous. }\end{array}$ & 13.6 & 13.4 & 0.31 & 0.86 & 1.18 & 731 & 25.45 & 0.79 & 3.0 & 0.18 & 737 & 42.4 & 43.4 & 43 & 7.4 \\
\hline
\end{tabular}


TABLE 1 - Continued

\begin{tabular}{|c|c|c|c|c|c|c|c|c|c|c|c|c|c|c|c|c|c|c|}
\hline $\begin{array}{l}\text { Sample } \\
\text { Desig- } \\
\text { nation }\end{array}$ & $\begin{array}{l}\text { Depth } \\
\text { (m) }\end{array}$ & Age & Description & $\mathrm{Na}^{\mathrm{a}}$ & $\mathrm{Na}^{\mathrm{b}}$ & K & $\mathrm{Ca}$ & $\mathrm{Mg}$ & $\begin{array}{c}\text { Total } \\
\text { Cations } \\
\text { (meq/kg) }\end{array}$ & $\mathrm{Cl}$ & $\mathrm{SO}_{4}$ & $\begin{array}{c}\text { Alk. } \\
(\mathrm{meq} / \mathrm{kg})\end{array}$ & $\mathrm{HCO}_{3}{ }^{\mathrm{c}}$ & $\begin{array}{c}\text { Total } \\
\text { Anions } \\
\text { (meq } / \mathrm{kg})\end{array}$ & Sum $^{d}$ & $\begin{array}{l}\text { Refrac- } \\
\text { tometer }\end{array}$ & $\begin{array}{l}\mathrm{H}_{2} \mathrm{O} \\
(\% \circ)^{\mathrm{e}}\end{array}$ & $\mathrm{pH}$ \\
\hline $140-4-3$ & 374 & $\begin{array}{l}\text { Middle } \\
\text { Eocene }\end{array}$ & $\begin{array}{l}\text { Varicolored gray silty } \\
\text { clay, interbedded layers. }\end{array}$ & 13.6 & 13.6 & 0.26 & 0.89 & 1.19 & 739 & 25.92 & 0.30 & 4.1 & 0.25 & 741 & 42.4 & 43.4 & 42 & 7.5 \\
\hline $6-\mathrm{CC}$ & 519 & $\begin{array}{l}\text { Paleocene } \\
\text { to Late } \\
\text { Cretaceous }\end{array}$ & $\begin{array}{l}\text { Dark olive gray silicified } \\
\text { claystone. }\end{array}$ & 15.3 & 15.2 & 0.25 & 0.85 & 1.22 & 811 & 28.44 & 0.48 & 2.6 & 0.16 & 815 & 46.7 & 47.3 & 39 & 7.5 \\
\hline $8-2$ & 647 & Unknown & $\begin{array}{l}\text { Dark greenish gray clay, } \\
\text { very firm. }\end{array}$ & 17.4 & 17.2 & 0.20 & 1.32 & 1.07 & 907 & 32.17 & 0.34 & 2.6 & 0.16 & 917 & 52.7 & 52.8 & 38 & 7.5 \\
\hline \multicolumn{19}{|c|}{ Hole $141\left(19^{\circ} 2\right.$} \\
\hline $141-1-6$ & 13 & $\begin{array}{l}\text { Early } \\
\text { Pleistocene }\end{array}$ & $\begin{array}{l}\text { Varicolored gray and } \\
\text { brown foram-nanno chalk } \\
\text { ooze }\end{array}$ & 10.9 & 10.8 & 0.40 & 0.44 & 1.25 & 604 & 19.67 & 2.43 & 3.3 & 0.20 & 608 & 35.3 & 35.5 & 43 & 7.4 \\
\hline $2-5$ & 20 & $\begin{array}{l}\text { Late } \\
\text { Pliocene }\end{array}$ & $\begin{array}{l}\text { Varicolored gray and } \\
\text { brown foram-nanno } \\
\text { chalk ooze. }\end{array}$ & 10.9 & 10.7 & 0.39 & 0.44 & 1.24 & 602 & 19.68 & 2.50 & 3.5 & 0.21 & 610 & 35.4 & 35.2 & 41 & 7.4 \\
\hline $3-6$ & 31 & $\begin{array}{l}\text { Middle } \\
\text { Pliocene }\end{array}$ & $\begin{array}{l}\text { Varicolored gray and } \\
\text { brown foram-nanno } \\
\text { chalk ooze. }\end{array}$ & 10.9 & 10.7 & 0.39 & 0.46 & 1.22 & 601 & 19.63 & 2.40 & 3.4 & 0.21 & 606 & 35.2 & 35.2 & 41 & 7.4 \\
\hline $4-4$ & 36 & $\begin{array}{l}\text { "Late } \\
\text { Early" } \\
\text { Pliocene }\end{array}$ & $\begin{array}{l}\text { Light gray, yellowish } \\
\text { gray and white foram- } \\
\text { nanno chalk ooze. }\end{array}$ & 11.0 & 10.7 & 0.39 & 0.46 & 1.21 & 599 & 19.71 & 2.46 & 3.7 & 0.23 & 610 & 34.5 & 35.2 & 36 & 7.5 \\
\hline $5-6$ & 49 & $\begin{array}{l}\text { "Middle } \\
\text { Early" } \\
\text { Pliocene }\end{array}$ & $\begin{array}{l}\text { White and light yellow- } \\
\text { ish gray foram-nanno } \\
\text { chalk ooze, slightly clayey. }\end{array}$ & 10.9 & 10.7 & 0.38 & 0.50 & 1.19 & 598 & 19.69 & 2.32 & 3.0 & 0.18 & 607 & 35.2 & 35.2 & 35 & 7.4 \\
\hline $7-6$ & 87 & $\begin{array}{l}\text { Early } \\
\text { Pliocene }\end{array}$ & $\begin{array}{l}\text { Yellowish brown silty } \\
\text { clay, slightly zeolitic. }\end{array}$ & 10.8 & 10.4 & 0.30 & 0.57 & 1.14 & 584 & 19.47 & 2.28 & 2.4 & 0.14 & 598 & 34.7 & 34.6 & 35 & 6.8 \\
\hline 8-CC & 123 & Unknown & $\begin{array}{l}\text { Brown to dark grayish } \\
\text { brown clay, slightly } \\
\text { zeolitic. }\end{array}$ & 10.8 & 10.6 & 0.31 & 0.67 & 1.08 & 592 & 19.69 & 2.05 & 1.7 & 0.07 & 599 & 34.7 & 35.2 & 43 & 6.8 \\
\hline $9-5$ & 197 & Unknown & $\begin{array}{l}\text { Reddish brown zeolitic } \\
\text { silty clay. }\end{array}$ & 10.2 & 10.1 & 0.17 & 1.18 & 0.80 & 568 & 19.35 & 1.31 & 0.7 & 0.04 & 574 & 33.1 & 33.0 & 30 & 6.8 \\
\hline \multicolumn{19}{|c|}{ Hole $142\left(3^{\circ} 22.2^{\prime} \mathrm{N}, 42^{\circ} 23.5^{\prime} \mathrm{W}\right.$, water depth $4372 \mathrm{~m}$, Ceara Abyssal Plain) } \\
\hline $142-1-6$ & 115 & Pleistocene & $\begin{array}{l}\text { Very dark gray to black } \\
\text { and olive gray to olive } \\
\text { black terrigenous silty } \\
\text { sand and silty clay. }\end{array}$ & 10.6 & 10.6 & 0.32 & 0.24 & 0.81 & 547 & 19.32 & 0.05 & 4.3 & 0.26 & 550 & 31.6 & 31.4 & 33 & 7.8 \\
\hline $2-4$ & 214 & $\begin{array}{l}\text { Early } \\
\text { Pleistocene }\end{array}$ & $\begin{array}{l}\text { Olive gray terrigenous } \\
\text { silty sand and gray slightly } \\
\text { silty calcareous mud. }\end{array}$ & 10.7 & 10.5 & 0.25 & 0.30 & 0.73 & 538 & 19.28 & $<0.01$ & 2.9 & 0.17 & 546 & 31.4 & 31.9 & 28 & 7.7 \\
\hline $3-\mathrm{CC}$ & 311 & $\begin{array}{l}\text { Early } \\
\text { Pleistocene }\end{array}$ & $\begin{array}{l}\text { Gray and dark gray silty } \\
\text { clay. }\end{array}$ & 10.9 & 10.6 & 0.22 & 0.30 & 0.71 & 541 & 19.48 & 0.05 & 1.9 & 0.12 & 552 & 31.8 & 31.4 & 21 & 7.6 \\
\hline $4-3$ & 380 & Pliocene & $\begin{array}{l}\text { Varicolored gray foram- } \\
\text { nanno chalk ooze. }\end{array}$ & 11.1 & 11.0 & 0.25 & 0.26 & 0.88 & 568 & 19.89 & 0.61 & 2.3 & 0.14 & 576 & 33.1 & 33.0 & 27 & 6.8 \\
\hline $5-\mathrm{CC}$ & 439 & Pliocene & $\begin{array}{l}\text { Light gray calcareous } \\
\text { clay and foram sand. }\end{array}$ & 10.8 & 10.8 & 0.24 & 0.33 & 0.89 & 564 & 19.61 & 0.45 & 3.2 & 0.20 & 565 & 32.5 & 33.0 & - & 7.5 \\
\hline
\end{tabular}


TABLE 1 - Continued

\begin{tabular}{|c|c|c|c|c|c|c|c|c|c|c|c|c|c|c|c|c|c|c|}
\hline $\begin{array}{l}\text { Sample } \\
\text { Desig- } \\
\text { nation }\end{array}$ & $\begin{array}{l}\text { Depth } \\
\text { (m) }\end{array}$ & Age & Description & $\mathrm{Na}^{\mathrm{a}}$ & $\mathrm{Na}^{\mathrm{b}}$ & $\mathrm{K}$ & $\mathrm{Ca}$ & $\mathrm{Mg}$ & $\begin{array}{l}\text { Total } \\
\text { Cations } \\
\text { (meq/kg) }\end{array}$ & $\mathrm{Cl}$ & $\mathrm{SO}_{4}$ & $\begin{array}{l}\text { Alk. } \\
\text { (meq/kg) }\end{array}$ & $\mathrm{HCO}_{3}{ }^{\mathrm{c}}$ & $\begin{array}{l}\text { Total } \\
\text { Anions } \\
\text { (meq/kg) }\end{array}$ & Sum $^{\mathrm{d}}$ & $\begin{array}{l}\text { Refrac- } \\
\text { tometer }\end{array}$ & $\begin{array}{l}\mathrm{H}_{2} \mathrm{O} \\
(\% \circ)^{\mathrm{e}}\end{array}$ & $\mathrm{pH}$ \\
\hline $142-6-\mathrm{CC}$ & 467 & $\begin{array}{l}\text { Late } \\
\text { Miocene }\end{array}$ & $\begin{array}{l}\text { Brown to olive gray } \\
\text { nanno marl mud, light } \\
\text { gray nanno chalk ooze, } \\
\text { allochthonous silty } \\
\text { foram sands. }\end{array}$ & 10.9 & 10.8 & 0.27 & 0.36 & 0.96 & 574 & 19.76 & 0.89 & 2.5 & 0.15 & 578 & 33.3 & 33.0 & 22 & 7.4 \\
\hline $7-6$ & 496 & $\begin{array}{l}\text { Late } \\
\text { Miocene }\end{array}$ & $\begin{array}{l}\text { Pale yellow, v. pale } \\
\text { brown, light gray } \\
\text { foram-nanno marl to } \\
\text { chalk ooze. }\end{array}$ & 11.0 & 10.8 & 0.29 & 0.40 & 0.99 & 580 & 19.89 & 1.13 & 3.6 & 0.22 & 588 & 33.9 & 34.1 & 18 & 7.2 \\
\hline $8-\mathrm{CC}$ & 548 & $\begin{array}{l}\text { "Late } \\
\text { Early" } \\
\text { Miocene }\end{array}$ & Bluish gray clay. & 9.8 & 9.7 & 0.19 & 0.88 & 1.04 & 558 & 19.04 & 1.09 & - & - & 559 & 32.0 & - & - & 7.6 \\
\hline $9-1$ & 587 & $\begin{array}{l}\text { Early } \\
\text { Miocene }\end{array}$ & $\begin{array}{l}\text { Varicolored gray nanno } \\
\text { marl mud, indurated. }\end{array}$ & 10.5 & 10.2 & 0.26 & 1.02 & 0.91 & 577 & 19.68 & 1.42 & 5.8 & 0.36 & 590 & 34.1 & 34.4 & 21 & 7.1 \\
\hline Hole 144, & , 144A, & 144B $\left(9^{\circ} \quad 27\right.$. & $2^{\prime} \mathrm{N}, 54^{\circ} \quad 20.5^{\prime} \mathrm{W}$, water de & epth 2 & $2957 \mathrm{n}$ & $n$, nort & thern $\mathrm{f}$ & lank of & Demerara & Rise) & & & & & & & & \\
\hline \multicolumn{4}{|c|}{ Surface ocean water } & 10.6 & 10.3 & 0.38 & 0.39 & 1.25 & 582 & 18.92 & 2.64 & 4.0 & 0.24 & 593 & 34.4 & 34.1 & - & - \\
\hline $144 B-1-6$ & 8 & $\begin{array}{l}\text { Middle } \\
\text { Oligocene }\end{array}$ & $\begin{array}{l}\text { Pale yellow foram-nanno } \\
\text { chalk. }\end{array}$ & 10.7 & 10.4 & 0.52 & 0.39 & 1.23 & 588 & 19.20 & 2.60 & 2.9 & 0.18 & 598 & 34.8 & 35.2 & 39 & 7.4 \\
\hline $2-5$ & 16 & $\begin{array}{l}\text { Middle } \\
\text { Oligocene }\end{array}$ & $\begin{array}{l}\text { Pale yellow foram-nanno } \\
\text { chalk slightly siliceous. }\end{array}$ & 11.0 & 10.8 & 0.51 & 0.42 & 1.25 & 607 & 19.73 & 2.67 & 3.3 & 0.20 & 615 & 35.8 & 35.5 & 40 & 7.3 \\
\hline $144 \mathrm{~A}-1-2$ & 22 & $\begin{array}{l}\text { Middle } \\
\text { Oligocene }\end{array}$ & $\begin{array}{l}\text { Pale yellow green foram- } \\
\text { nanno chalk. }\end{array}$ & 11.2 & 11.0 & 0.55 & 0.42 & 1.21 & 611 & 20.06 & 2.44 & 3.6 & 0.22 & 620 & 36.1 & 35.8 & 40 & 7.5 \\
\hline $144 \mathrm{~B}-3-6$ & 35 & $\begin{array}{l}\text { Early } \\
\text { Oligocene }\end{array}$ & $\begin{array}{l}\text { Pale yellowish green to } \\
\text { pale green foram-nanno } \\
\text { chalk. }\end{array}$ & 11.3 & 11.1 & 0.53 & 0.42 & 1.18 & 615 & 20.24 & 2.38 & 2.2 & 0.13 & 623 & 36.2 & 36.3 & 40 & 7.8 \\
\hline $144 \mathrm{~A}-2-6$ & 46 & $\begin{array}{l}\text { Early } \\
\text { Oligocene }\end{array}$ & $\begin{array}{l}\text { Pale yellowish green } \\
\text { foram-nanno chalk. }\end{array}$ & 11.5 & 11.3 & 0.52 & 0.43 & 1.20 & 627 & 20.62 & 2.34 & 2.8 & 0.17 & 633 & 36.8 & 36.3 & 40 & 7.4 \\
\hline $144-1-6$ & 64 & $\begin{array}{l}\text { Late Middle } \\
\text { Eocene }\end{array}$ & $\begin{array}{l}\text { Light greenish gray foram- } \\
\text { nanno-rad marl/ooze. }\end{array}$ & 11.5 & 11.4 & 0.41 & 0.49 & 1.19 & 630 & 20.67 & 2.12 & 4.8 & 0.29 & 632 & 36.7 & 36.6 & - & - \\
\hline $144 \mathrm{~A}-3-4$ & 144 & $\begin{array}{l}\text { Late } \\
\text { Paleocene }\end{array}$ & $\begin{array}{l}\text { Greenish gray zeolitic } \\
\text { foram-nanno marl/chalk. }\end{array}$ & 13.6 & 13.4 & 0.34 & 0.75 & 1.02 & 710 & 25.24 & 0.40 & 0.9 & 0.05 & 721 & 41.4 & 41.8 & 18 & 7.0 \\
\hline $144-3-2$ & 164 & $\begin{array}{l}\text { Early Mae- } \\
\text { strichtian } \\
\text { to Late } \\
\text { Campanian }\end{array}$ & $\begin{array}{l}\text { Light greenish gray marl } \\
\text { mudstone. }\end{array}$ & 13.6 & 13.4 & 0.32 & 0.76 & 0.99 & 709 & 24.94 & 0.56 & 2.1 & 0.13 & 717 & 41.3 & 41.2 & - & - \\
\hline \multicolumn{2}{|c|}{$144 \mathrm{~A}-4-\mathrm{CC} 180$} & $\begin{array}{l}\text { Early Mae- } \\
\text { strichtian } \\
\text { to Late } \\
\text { Campanian }\end{array}$ & $\begin{array}{l}\text { Greenish gray zeolitic } \\
\text { nanno marl. }\end{array}$ & 14.5 & 14.4 & 0.31 & 0.91 & 0.94 & 758 & 27.00 & 0.01 & 0.8 & 0.05 & 762 & 43.7 & 44.0 & 25 & 6.9 \\
\hline $5-\mathrm{CC}$ & C 189 & Senonian & $\begin{array}{l}\text { Olive green foram glaucon- } \\
\text { itic and phosphoritic } \\
\text { limestone. }\end{array}$ & 14.8 & 14.7 & 0.31 & 0.97 & 0.86 & 766 & 27.17 & 0.15 & 1.8 & 0.11 & 771 & 44.4 & 45.1 & - & 7.0 \\
\hline 6-CC & C 197 & Senonian & $\begin{array}{l}\text { Olive black zeolitic cal- } \\
\text { careous carbonaceous } \\
\text { shale. }\end{array}$ & 15.2 & 15.1 & 0.29 & 1.09 & 0.84 & 786 & 27.94 & 0.12 & 1.9 & 0.11 & 792 & 45.6 & 45.9 & - & 6.9 \\
\hline
\end{tabular}


TABLE 1 - Continued

\begin{tabular}{|c|c|c|c|c|c|c|c|c|c|c|c|c|c|c|c|c|c|c|}
\hline $\begin{array}{l}\text { Sample } \\
\text { Desig- } \\
\text { nation }\end{array}$ & $\begin{array}{l}\text { Depth } \\
(\mathrm{m})\end{array}$ & Age & Description & $\mathrm{Na}^{\mathrm{a}}$ & $\mathrm{Na}^{\mathrm{b}}$ & $\mathrm{K}$ & $\mathrm{Ca}$ & $\mathrm{Mg}$ & $\begin{array}{c}\text { Total } \\
\text { Cations } \\
\text { (meq } / \mathrm{kg})\end{array}$ & $\mathrm{Cl}$ & $\mathrm{SO}_{4}$ & $\underset{(\mathrm{meq} / \mathrm{kg})}{\text { Alk. }}$ & $\mathrm{HCO}_{3}{ }^{\mathrm{c}}$ & $\begin{array}{c}\text { Total } \\
\text { Anions } \\
(\mathrm{meq} / \mathrm{kg})\end{array}$ & Sum $^{d}$ & $\begin{array}{l}\text { Refrac- } \\
\text { tometer }\end{array}$ & $\begin{array}{l}\mathrm{H}_{2} \mathrm{O} \\
(\% \circ)^{\mathrm{e}}\end{array}$ & $\mathrm{pH}$ \\
\hline $144-4-3$ & 216 & $\begin{array}{l}\text { Cenomanian } \\
\text { to Early } \\
\text { Turonian }\end{array}$ & $\begin{array}{l}\text { Dark olive gray carbon- } \\
\text { aceous clay. }\end{array}$ & 15.1 & 14.7 & 0.28 & 1.03 & 0.93 & 776 & 27.92 & 0.09 & 1.9 & 0.12 & 791 & 45.5 & 46.2 & - & - \\
\hline $5-\mathrm{CC}$ & 270 & $\begin{array}{l}\text { Late Albian } \\
\text { to Early } \\
\text { Turonian }\end{array}$ & $\begin{array}{l}\text { Olive gray marl semi- } \\
\text { indurated to plastic. }\end{array}$ & - & 13.5 & 0.18 & 0.88 & 1.10 & 726 & $(25.28)^{f}$ & 0.50 & 2.5 & 0.15 & (726) & 41.6 & - & - & - \\
\hline
\end{tabular}

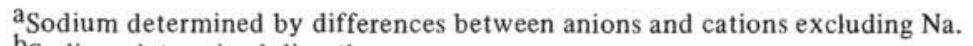

$\mathrm{b}_{\text {Sodium determined directly. }}$

$\mathrm{C}_{\mathrm{HCO}}$ is calculated from total alkalinity, assuming this is entirely due to bicarbonate ion.

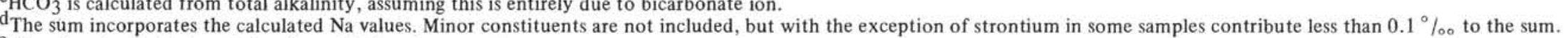

$\mathrm{e}_{\mathrm{pH}}$ and water content are taken from shipboard summaries.

$\mathrm{f}_{\mathrm{Cl}}^{\mathrm{pH} \text { determined by difference in the absence of an analytical value. }}$ 
TABLE 2

Minor Constituents. Concentrations in $\mathrm{mg} / \mathrm{kg}$ (ppm)

\begin{tabular}{|c|c|c|c|c|c|c|c|c|}
\hline $\begin{array}{l}\text { Sample } \\
\text { Designation }\end{array}$ & $\begin{array}{l}\text { Depth } \\
(\mathrm{m})\end{array}$ & Age & Description & B & $\mathrm{Sr}$ & $\mathrm{Ba}$ & $\mathrm{Si}(\mathrm{col} .)^{\mathrm{a}}$ & $\mathrm{Si}(\text { spec.) })^{\mathrm{b}}$ \\
\hline \multicolumn{9}{|l|}{ Hole 135} \\
\hline $135-1-2$ & 3 & Pleistocene & $\begin{array}{l}\text { Varicolored light brownish gray } \\
\text { nanno chalk ooze. }\end{array}$ & $?$ & 10.7 & $<0.5$ & 6.2 & $<5.0$ \\
\hline $2-6$ & 82 & Late Miocene & $\begin{array}{l}\text { Light gray nanno chalk ooze, } \\
\text { some black streaks. }\end{array}$ & 4 & 33.0 & $<0.5$ & 8.2 & $<5.0$ \\
\hline $4-2$ & 260 & Middle Miocene & $\begin{array}{l}\text { Varicolored light gray nanno } \\
\text { chalk ooze. }\end{array}$ & 6 & 61.0 & $<0.5$ & 6.2 & 14.0 \\
\hline $7-3$ & 435 & Lower Campanian & $\begin{array}{l}\text { Green, olive gray, dark brown } \\
\text { silty mudstone, sand and "red } \\
\text { clay" layers, intercalated. }\end{array}$ & 5 & 58.0 & $<0.5$ & 4.1 & $\leqslant 5.0$ \\
\hline
\end{tabular}

\section{Hole 136}

\begin{tabular}{|c|c|c|c|c|c|c|c|c|}
\hline \multicolumn{5}{|c|}{ Surface ocean water } & \multirow{2}{*}{$\begin{array}{r}7.6 \\
80.0\end{array}$} & \multirow{2}{*}{$\begin{array}{l}<0.5 \\
<0.5\end{array}$} & \multirow{2}{*}{$\overline{5.0}$} & \multirow{2}{*}{$\leqslant 5.0$} \\
\hline $136-1-5$ & 136 & Early Pliocene & $\begin{array}{l}\text { Very pale orange nanno chalk } \\
\text { ooze. }\end{array}$ & 5 & & & & \\
\hline $2-6$ & 224 & $\begin{array}{l}\text { Middle Early } \\
\text { Miocene }\end{array}$ & $\begin{array}{l}\text { Very pale orange and pale brown } \\
\text { nanno chalk ooze. }\end{array}$ & 6 & 46.0 & $<0.5$ & 13.7 & 10.3 \\
\hline $3-4$ & 238 & Early Miocene & $\begin{array}{l}\text { Vary pale brown to pale brown } \\
\text { nanno chalk ooze with light gray } \\
\text { lenses and mottles. }\end{array}$ & 6 & 42.0 & $<0.5$ & 9.1 & 8.3 \\
\hline $4-3$ & 248 & Early Miocene (?) & $\begin{array}{l}\text { Brown and grayish brown silty } \\
\text { clay }\end{array}$ & 5 & 41.0 & $<0.5$ & 20.0 & 22.0 \\
\hline $6-1$ & 264 & $\begin{array}{l}\text { Santonian to } \\
\text { Campanian }\end{array}$ & $\begin{array}{l}\text { Brownish red and pale yellowish } \\
\text { brown silty clay. }\end{array}$ & 8 & 36.0 & $<0.5$ & 7.0 & 6.4 \\
\hline $8-2$ & 282 & Unknown & Reddish brown marl mud & 5 & 33.0 & $<0.5$ & 12.8 & 13.2 \\
\hline \multicolumn{9}{|l|}{ Hole 137} \\
\hline \multicolumn{4}{|c|}{ Surface ocean water } & - & 7.3 & $<0.5$ & - & $<5.0$ \\
\hline $137-1-5$ & 58 & Unknown & $\begin{array}{l}\text { Yellowish brown and dark } \\
\text { grayish brown silty clay. }\end{array}$ & 6 & 8.0 & $<0.5$ & 10.2 & 5.7 \\
\hline $2-1$ & 100 & Unknown & Brick red silty zeolitic clay. & 4 & 8.6 & $<0.5$ & 10.0 & 7.8 \\
\hline $2-\mathrm{CC}$ & 104 & Unknown & Brown clay. & 4 & 10.1 & $<0.5$ & 18.3 & 21.0 \\
\hline $3-6$ & 143 & Unknown & $\begin{array}{l}\text { Varicolored brown clay, slightly } \\
\text { zeolitic }\end{array}$ & 5 & 10.5 & $<0.5$ & 13.4 & 14.9 \\
\hline $4-2$ & 168 & Unknown & $\begin{array}{l}\text { Reddish brown clay, slightly } \\
\text { silty, zeolitic. }\end{array}$ & 4 & 10.5 & $<0.5$ & 17.3 & 23.0 \\
\hline $6-\mathrm{CC}$ & 225 & $\begin{array}{l}\text { Turonian (?) } \\
\text { Coniacian }\end{array}$ & Reddish brown clay, zeolitic & 2 & 10.1 & $<0.5$ & 26.0 & 24.0 \\
\hline 7-CC & 265 & $\begin{array}{l}\text { Cenomanian-Early } \\
\text { Turonian }\end{array}$ & $\begin{array}{l}\text { Varicolored alternations of dark } \\
\text { carbonaceous clay, nanno marl } \\
\text { ooze and nanno chalk ooze. }\end{array}$ & 2 & 19.1 & $<0.5$ & 2.6 & $<5.0$ \\
\hline $8-\mathrm{CC}$ & 274 & Late Cenomanian & $\begin{array}{l}\text { Dark gray foram-nanno chalk } \\
\text { ooze. }\end{array}$ & 4 & 16.0 & $<0.5$ & 7.1 & $<5.0$ \\
\hline $9-5$ & 282 & Late Cenomanian & $\begin{array}{l}\text { Pale red and light greenish gray } \\
\text { nanno marl ooze to chalk ooze. }\end{array}$ & 2 & 19.5 & $<0.5$ & 4.3 & $<5.0$ \\
\hline $10-5$ & 287 & Cenomanian & $\begin{array}{l}\text { Reddish brown and greenish } \\
\text { gray nanno marl to chalk ooze. }\end{array}$ & 4 & 14.9 & $<0.5$ & 8.7 & $<5.0$ \\
\hline $16-5$ & 381 & Late Albian & $\begin{array}{l}\text { Gray and brown nanno marl ooze } \\
\text { and foram-nanno chalk ooze. }\end{array}$ & 2 & 21.1 & $17.6(?)$ & 5.9 & $<5.0$ \\
\hline \multicolumn{9}{|l|}{ Hole 138} \\
\hline $138-1-5$ & 58 & Early Miocene & $\begin{array}{l}\text { Pale yellowish green and pale } \\
\text { yellowish brown clay and sand. }\end{array}$ & 7 & 7.7 & $<0.5$ & 18.5 & 28.0 \\
\hline $2-5$ & 116 & Oligocene & $\begin{array}{l}\text { Greenish gray and olive gray } \\
\text { silty clay. }\end{array}$ & 4 & 9.0 & $<0.5$ & 28.0 & 44.0 \\
\hline \multicolumn{9}{|l|}{ Hole 139} \\
\hline $139-1-2$ & 116 & Early Pliocene & $\begin{array}{l}\text { Light olive gray to light gray } \\
\text { foram nanno chalk ooze } ; \mathrm{H}_{2} \mathrm{~S} \\
\text { odor }\end{array}$ & 5 & 45.0 & $<0.5$ & 25.0 & 15.9 \\
\hline
\end{tabular}


TABLE 2 - Continued

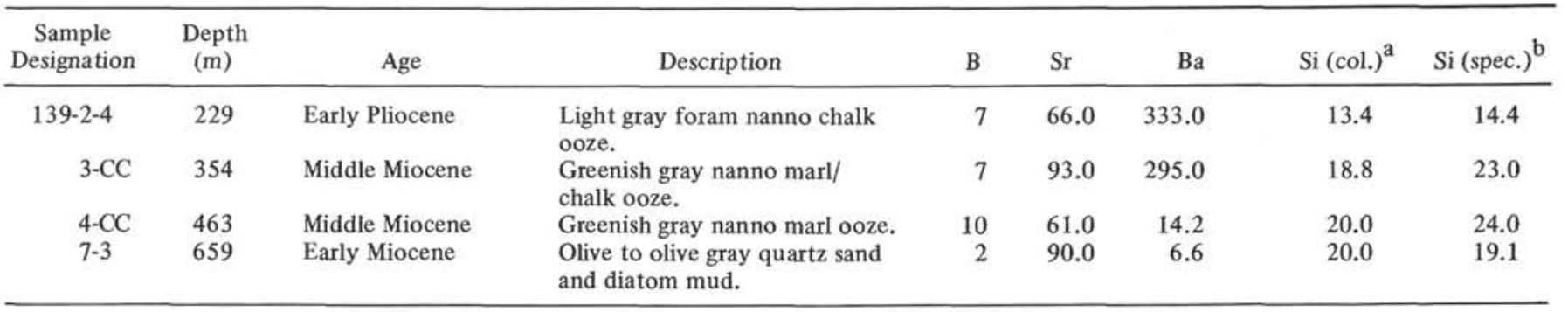

\section{Holes 140 \& 140A}

\begin{tabular}{|c|c|c|c|c|c|c|c|c|}
\hline $140-1-6$ & 96 & Late Pliocene & $\begin{array}{l}\text { Very light yellowish gray foram- } \\
\text { nanno chalk ooze. }\end{array}$ & 4 & 17.7 & $<0.5$ & 3.4 & $<5.0$ \\
\hline $2-6$ & 209 & Early Miocene & $\begin{array}{l}\text { Grayish olive diatom mud/ooze } \\
\text { quartz bearing }\end{array}$ & 4 & 15.7 & 1.6 & 20.0 & 21.0 \\
\hline $140 A-2-6$ & 243 & Middle Eocene & $\begin{array}{l}\text { Varicolored green and gray clay, } \\
\text { slightly siliceous, firm }\end{array}$ & 4 & 19.1 & $<0.5$ & 24.0 & 26.0 \\
\hline $140-3-2$ & 313 & Middle Eocene & $\begin{array}{l}\text { Greenish gray silty clay slightly } \\
\text { siliceous }\end{array}$ & 3 & 24.0 & $<0.5$ & 20.0 & 8.6 \\
\hline $4-3$ & 374 & Middle Eocene & $\begin{array}{l}\text { Varicolored gray silty clay, } \\
\text { interbedded layers. }\end{array}$ & 7 & 26.0 & 1.4 & 18.0 & 18.4 \\
\hline 6-CC & 519 & $\begin{array}{l}\text { Paleocene to Late } \\
\text { Cretaceous }\end{array}$ & $\begin{array}{l}\text { Dark olive gray silicified } \\
\text { claystone. }\end{array}$ & 4 & 38.0 & 0.8 & 22.5 & 20.3 \\
\hline $6-2$ & 647 & Unknown & $\begin{array}{l}\text { Dark greenish gray clay, very } \\
\text { firm. }\end{array}$ & 3 & 37.0 & 1.7 & 5.2 & 5.9 \\
\hline
\end{tabular}

Hole 141

\begin{tabular}{|c|c|c|c|c|c|c|c|c|}
\hline $141-1-6$ & 13 & Early Pleistocene & $\begin{array}{l}\text { Varicolored gray and brown } \\
\text { foram-nanno chalk ooze. }\end{array}$ & 4 & 12.3 & $<0.5$ & 3.4 & $<5.0$ \\
\hline $2-5$ & 20 & Late Pliocene & $\begin{array}{l}\text { Varicolored gray and brown } \\
\text { foram-nanno chalk ooze. }\end{array}$ & 4 & 16.9 & $<0.5$ & 3.4 & $<5.0$ \\
\hline $3-6$ & 31 & Middle Pliocene & $\begin{array}{l}\text { Varicolored gray and brown } \\
\text { foram-nanno chalk ooze. }\end{array}$ & 6 & 11.8 & $<0.5$ & 4.3 & $<5.0$ \\
\hline $4-4$ & 36 & $\begin{array}{l}\text { "Late Early" } \\
\text { Pliocene }\end{array}$ & $\begin{array}{l}\text { Light gray, yellowish gray and } \\
\text { white foram-nanno chalk ooze. }\end{array}$ & 8 & 16.1 & $<0.5$ & 2.1 & $<5.0$ \\
\hline $5-6$ & 49 & $\begin{array}{l}\text { "Middle Early" } \\
\text { Pliocene }\end{array}$ & $\begin{array}{l}\text { White and light yellowish gray } \\
\text { foram-nanno chalk ooze, } \\
\text { slightly clayey. }\end{array}$ & 7 & 14.4 & $<0.5$ & 3.5 & 10.3 \\
\hline $7-6$ & 87 & Early Pliocene & $\begin{array}{l}\text { Yellowish brown silty clay, } \\
\text { slightly zeolitic. }\end{array}$ & 9 & 16.2 & $<0.5$ & 6.8 & $<5.0$ \\
\hline $8-\mathrm{CC}$ & 123 & Unknown & $\begin{array}{l}\text { Brown to dark grayish brown } \\
\text { clay, slightly zeolitic. }\end{array}$ & 8 & 15.5 & $<0.5$ & 13.8 & 23.0 \\
\hline $9-5$ & 197 & Unknown & $\begin{array}{l}\text { Reddish brown zeolitic silty } \\
\text { clay. }\end{array}$ & 8 & 17.1 & $<0.5$ & 9.9 & 7.8 \\
\hline \multicolumn{9}{|l|}{ Hole 142} \\
\hline $142-1-6$ & 115 & Pleistocene & $\begin{array}{l}\text { Very dark gray to black and olive } \\
\text { gray to olive black terrigenous } \\
\text { silty sand and silty clay. }\end{array}$ & 4 & 3.9 & 7.9 & 4.7 & $<5.0$ \\
\hline $2-4$ & 214 & Early Pleistocene & $\begin{array}{l}\text { Olive gray terrigenous silty sand } \\
\text { and gray slightly silty calcareous } \\
\text { mud. }\end{array}$ & 4 & 8.6 & 6.7 & 6.5 & $<5.0$ \\
\hline $3-\mathrm{CC}$ & 311 & Early Pleistocene & Gray and dark gray silty clay. & 5 & 34.0 & 8.36 & 2.1 & $<5.0$ \\
\hline $4-3$ & 380 & Pliocene & $\begin{array}{l}\text { Varicolored gray foram-nanno } \\
\text { chalk ooze. }\end{array}$ & 7 & 63.0 & 0.79 & 4.6 & $<5.0$ \\
\hline $5-\mathrm{CC}$ & 439 & Pliocene & $\begin{array}{l}\text { Light gray calcareous clay and } \\
\text { foram sand. }\end{array}$ & 14 & 44.0 & $<0.5$ & 2.5 & $<5.0$ \\
\hline $6-\mathrm{CC}$ & 467 & Late Miocene & $\begin{array}{l}\text { Brown to olive gray nanno marl } \\
\text { mud, light gray nanno chalk } \\
\text { ooze, allochthonous silty foram } \\
\text { sands. }\end{array}$ & 5 & 80.0 & $<0.5$ & 2.0 & 5.2 \\
\hline $7-6$ & 496 & Late Miocene & $\begin{array}{l}\text { Pale yellow, v. pale brown, light } \\
\text { gray foram-nanno marl to chalk } \\
\text { ooze. }\end{array}$ & 5 & 94.0 & $<0.5$ & 7.5 & $<5.0$ \\
\hline $8-\mathrm{CC}$ & 548 & $\begin{array}{l}\text { "Late Early" } \\
\text { Miocene }\end{array}$ & Bluish gray clay. & - & - & - & 8.2 & - \\
\hline $9-1$ & 587 & Early Miocene & $\begin{array}{l}\text { Varicolored gray nanno marl } \\
\text { mud, indurated. }\end{array}$ & 4 & 120.0 & $<0.5$ & 23.4 & 21.0 \\
\hline
\end{tabular}


TABLE 2 - Continued

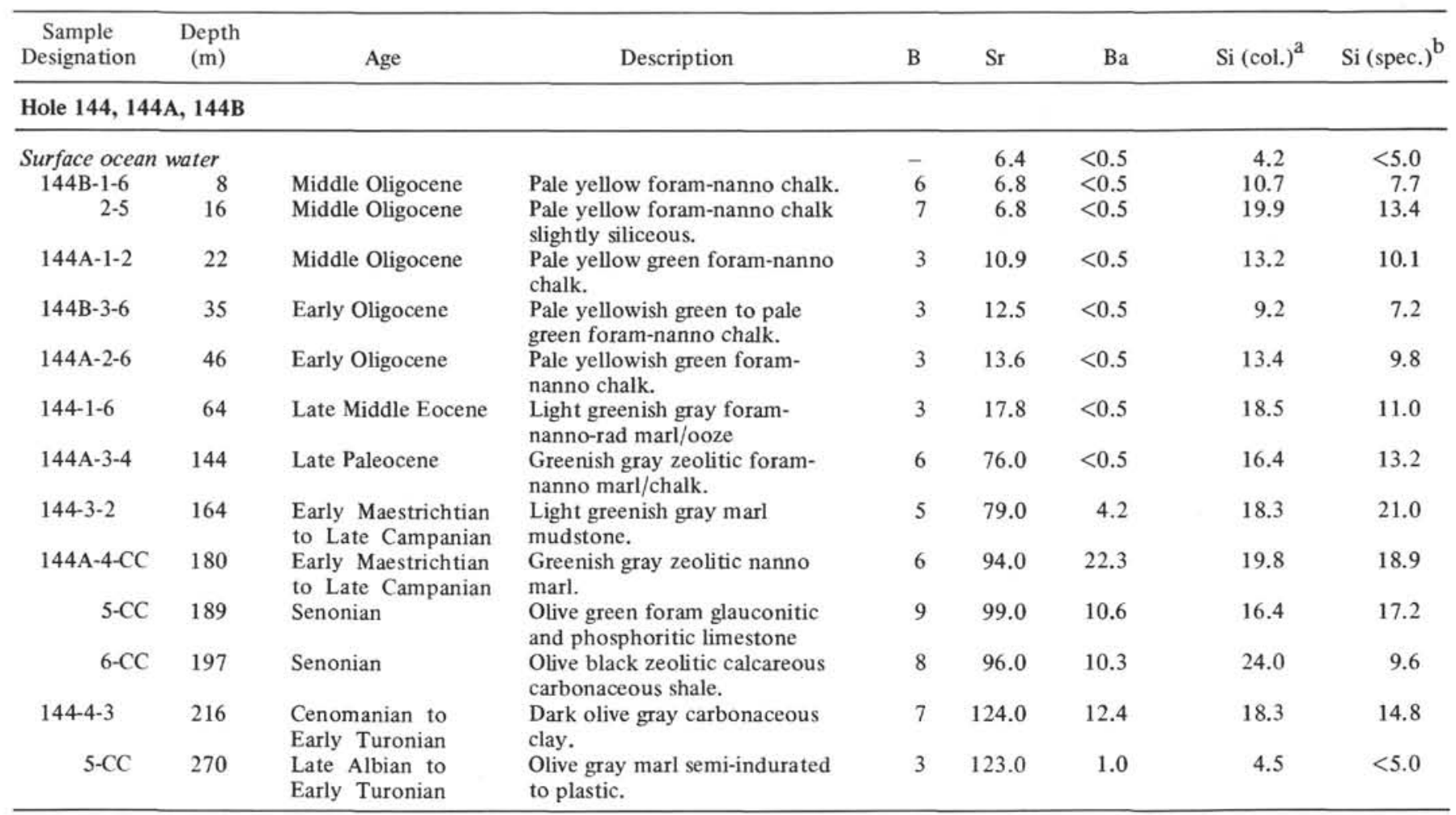

a $($ col. $)=$ Colorimetric determination.

(spec. $)=$ Emission spectrographic determination. 